\title{
How Feature Context alters Attentional Template Switching
}

\author{
Jan Tünnermann ${ }^{1}$, Leonardo Chelazzi ${ }^{2}$ \& Anna Schubö ${ }^{1}$ \\ ${ }^{1}$ Cognitive Neuroscience of Perception and Action, Department of Psychology, \\ Philipps-University Marburg, Germany. \\ ${ }^{2}$ Department of Neuroscience, Biomedicine and Movement Sciences, \\ University of Verona, Verona, Italy
}

(C) American Psychological Association, 2021. This paper is not the copy of
record and may not exactly replicate the authoritative document published in
the APA journal. Please do not copy or cite without author's permission. The
final article is available, upon publication, at: Journal of Experimental
Psychology: Human Perception and Performance; DOI: $10.1037 / x h p 0000951$

\section{Author Note}

Jan Tünnermann (Dhttps://orcid.org/0000-0003-1861-3115

Leonardo Chelazzi iDhttps://orcid.org/0000-0001-8566-0611

Anna Schubö (D) https://orcid.org/0000-0002-3274-1693

Correspondence concerning this article should be addressed to Jan Tünnermann, Department of

Psychology, Philipps-University Marburg, Gutenbergstraße 18, 35032 Marburg, Germany.

E-mail: jan.tuennermann@uni-marburg.de 


\begin{abstract}
In real-world tasks visual attention is rarely aimed at a single object. Humans rather "forage" the visual scene for information, dynamically switching attentional templates. Several visual search studies have found that observers often use suboptimal attentional control strategies, possibly to avoid effort. Here, we investigated with a foraging paradigm if observers' reluctance to switch between attentional templates increases with template specificity. To that end, we manipulated the feature context of displays in which participants "foraged" moving stimuli on a tablet-PC. Experiment $1(N=35)$ revealed a decline in switching tendency and foraging efficiency with increasing feature-space distance between target alternatives. Experiment $2(N=36)$ found even lower flexibility with distractor color close to, and strongest impairments with distractor color in between target colors. Our results demonstrate that visual information sampling is most flexible when broad (instead of very specific) templates and relational search strategies are possible (e.g., attending to "redder" objects), with implications for both attention research and applications, especially in visual-foraging-like tasks, such as baggage screening or medical image assessment.
\end{abstract}

Keywords: visual attention, attentional templates, attentional control, visual search, visual foraging

\title{
Public Significance Statement
}

Humans use various strategies when searching for multiple targets, for instance, in baggage screening, medical image assessment, or when picking berries from a bush. This study demonstrates that even slight changes to the appearance of objects can substantially alter selection preferences and search efficiency. Some situations are particularly harmful to efficient search. For instance, if the environments contain targets which require highly featurespecific templates, searchers lose their ability to flexibly switch between different target colors. Instead, they limit their selections to one target type over prolonged periods, and their search becomes slower. 


\section{Introduction}

Of all the visual information constantly reaching the brain, only a small fraction is relevant at any given moment. Optimally, the most relevant information is selected and integrated into our behavior, guiding eye movements or other actions, or recruiting a share of our limited cognitive resources for further processing. The mechanisms that orchestrate this filtering and prioritization are collectively known as selective visual attention. Visual attention is influenced by various stimulus factors, such as salience, task-relevance, scene structure, perceived value, and the history of prior searches (Wolfe \& Horowitz, 2017).

The present study investigates goal-directed attention guidance by assessing how observers switch between attentional templates while searching for multiple targets. Attentional templates are memory representations of search targets that facilitate the selection of elements in a scene with features matching (or similar to) those of the template (see Duncan, \& Humphreys, 1989). Single-cell recordings (Chelazzi et al., 1993, 1998), electrophysiological experiments (Carlisle et al., 2011), and behavioral experiments (e.g., Olivers et al., 2006) have demonstrated that attentional templates are stored in visual working memory (VWM; see also Olivers et al., 2011).

Different streams of research have demonstrated that attentional control can be flexibly adjusted depending on task requirements (cf. Irons \& Leber, 2016). For instance, Bacon \& Egeth (1994) showed that in visual search tasks observers can use a "singleton detection mode" to detect salient items in the display. When "singleton detection mode" was rendered inefficient by either introducing further targets of the same type (harming target feature uniqueness) or by introducing distractors with unique features, observer searched for specific features instead of salient items.

It has also been shown that the visual-attention system can tune target template specificity in more gradual terms. For instance, observers can "sharpen" their attentional templates to improve their selectiveness (Geng et al., 2017; Yu \& Geng, 2019), in line with the idea that attention toward specific features enhances neural processing of relevant stimuli (cf. Martinez-Trujillo \& Treue, 2004). However, the studies by Geng and colleagues also showed that this template sharpening is not necessarily homing in on the closest veridical representation of the target (see also Geng \& Witkowski, 2019). Instead, the sharpening seems to be asymmetric, bending tuning functions away from distractor features nearby in feature space. This 
connects to earlier research that also found such relational components in templates.

Navalpakkam \& Itti (2007; see also Kerzel, 2020) showed that tuning functions are shifted away from nontargets if targets and nontargets can be linearly separated in feature space. For instance, for orange targets among yellow nontargets, the template facilitates values even redder than the target, which still aids target selection, while making nontarget selection less likely. Becker (2010; Martin \& Becker, 2018; York \& Becker, 2020) put forward a relational account to explain such shifts, in which attention is guided towards the feature space direction in which the target differs from nontargets. In their experiments they showed that observers are most distracted not by elements most similar to the target but by those that differed most strongly from the nontargets in the same direction as the target.

As a common thread running through these studies, it seems that even though more specialized templates seem more helpful to guide attention to the target, observers seem to prefer less specialized templates, if possible. Irons \& Leber (2016) point out that participants often use singleton detection mode even though it makes them more sensitive to attention being captured by the distractor. Similarly, Becker et al. (2014) found observers performing relational search, again despite their ability of performing feature specific search and increased vulnerability to distraction. Yu and Geng (2019) also found sharpening to occur only when close-by distractor features made it necessary. These observations might hint that template specialization comes with a cost which the observer prefers to avoid. Whether this is the case also in more natural settings such as foraging, remains unclear. Crucially, this seems highly relevant for applications, for instance, in visualizations or human-machine interaction. In such domains one might be tempted to assume the human visual-attention system would always use the most specific templates to shield off distraction. However, the results discussed above suggest that observers might rather resort to the most general ones that seem sufficient most of the time.

Some researchers have suggested increased cognitive effort as the reason for apparently superior attentional strategies being avoided (Bacon \& Egeth, 1994; Irons \& Leber, 2016; Yu \& Geng., 2019). Adaptive choice visual search studies (Irons \& Leber, 2016; 2018; Bergmann et al., 2019) have provided some evidence for effort minimization in attentional control. In these studies, observers could choose one of two different targets. Over the course of the experiments, similarity of distractor and target features varied systematically: In "plateau" periods, one target was unique in color and the other resembled the distractors. In "transition" periods, trial by trial 
distractors became more similar to the target that was unique in the plateau and less similar to the other, originally inconspicuous, target. At the center of the transition period, both targets competed with an equal number of similar distractors. In the second half of the transition the originally inconspicuous target trial by trial became more unique until a plateau was reached in which the original roles had been reversed. Participants adapted to these regularities to some degree, favoring the targets less similar to the distractors. However, the point at which their preference flipped from one target to the other was typically not at the center of the transition but shifted to later trials. This has been interpreted as reluctance to updating attentional control settings and a sign of effort minimization.

The observations that show that observers tend to use more general attentional templates if the task permits and that they sometimes seem reluctant to update their attentional control settings leads to a question that has so far not been addressed and which is at the core of the present study: Is switching between more specific templates more costly or effortful than switching between more general ones? Traditional single-target visual search paradigms are not well suited to address this question because they are highly discontinuous. Such tasks with discrete presentation sequences may enable precise experimental control and potentially catch the visual system off guard, revealing parts of its inner workings. However, especially when it comes to the expression of attentional strategies, it seems important to investigate them under naturalistic conditions. For instance, the time between trials could be utilized for template management, hiding its impact on overall performance. From a more general perspective, Hommel (2009) warned about tailoring theories tightly toward typical laboratory tasks. $\mathrm{He}$ wrote: "Presenting carefully selected stimuli and measuring arbitrary responses to them provides many advantages, but real actions are commonly not driven by stimuli, not carried out to subserve meaningless goals, and not aimed at carrying out movements for their own sake" (p. 523). This assessment seems highly relevant for the issues related to attentional template switching.

The present study investigates attentional template switching within a visual foraging paradigm. Unlike traditional visual search tasks, in which participants search for a single (or very few) targets in successive displays, foraging tasks present observers with "patches" that contain multiple target instances from multiple target categories which the "foragers" collect in a behaviorally continuous manner (e.g., see Wolfe et al., 2018; Á. Kristjánsson et al., 2014). 
Foragers spent longer times in the patch selecting targets (among distractors) at a high rate, with many possibilities to switch or not to switch between different target categories. Potential switch costs in terms of time delays now become behaviorally relevant, as they accumulate and affect the overall intake rate. Moreover, switching or not switching has consequences for other behavioral components. For instance, skipping nearby targets of different types and instead going longer distances to pick those that match the current template leads to less efficient foraging paths and slower intake rates. To perform well, observers have to trade-off such consequences with costs related to template management. In short, the visual foraging paradigm provides a substrate for different attentional strategies. It is a naturalistic scenario with selections at high rates in which many aspects of template management become behaviorally relevant.

Visual foraging tasks have typically been applied to the question whether or not decisions to stay within a patch or leave it for another one follow optimal foraging theories. According to such theories, foragers decide in a manner that optimizes their intake rates over long sequences of patches. However, foraging tasks have also been applied to aspects of template switching. Á. Kristjánsson et al. (2014; see also T. Kristjánsson et al., 2018; T. Kristjánsson et al., 2020) had observers collect 40 targets among 40 distractors by tapping on them on an iPad. In the "feature foraging" condition, targets and distractors were defined within a single feature dimension (color): For instance, targets were red and blue (20 blue and 20 red disks) and distractors green and yellow (20 green and 20 yellow disks). In this setting, foragers switched flexibly between target alternatives and collected the elements with little effort. When foragers had to collect targets defined as conjunctions of two features (e.g. 20 green squares and 20 red circles among 20 green circles and 20 red squares), participants produced long same-type selection runs and experienced high switch costs (increased reaction times when switching targets). Same-type runs also occurred when observers foraged for as many as 64 pictorial stimuli (e.g., Wolfe et al., 2016).

In the present study we investigated the influence of template specificity on template switching in visual foraging tasks. Template specificity was manipulated by generating different "feature contexts". To that end, foraging displays contained target and distractor types with different color space distances and relationships. In our first experiment, we examined whether run-like selection behavior and switch costs occur when observers switch between targets that differ on a single feature dimension (color), and with highly distinct distractor features as not to 
encourage sharpening or relational tuning. We predicted that for targets that were very close in feature space, a single broad template might cover both targets, leading to frequent switches free of switch costs. For targets placed farther apart from each other in feature space, we expected that participants are more reluctant to switch between targets (producing more run-like selections) as switching between templates would entail switch costs. In our second experiment, we manipulated the feature context in such a way that targets were closer to each other and to the distractor in feature space, as to encourage template sharpening and relational template modifications (see Yu \& Geng, 2019). We predicted that the requirement of narrower templates leads to even more reluctance to switch, following the hypothesis that switching between more specific targets might be more costly or effortful. We also were interested in the nature of such switch costs. If avoiding template switches reduces overall path length and the time required to work through a patch, reduced template switching might occur in the service of performance maximization. If these foraging parameters indicate lower performance (taking potentially increased switch costs into account), reluctance to switch templates might rather be a consequence of effort minimization.

\section{Experiment 1}

Experiment 1 varied target dissimilarity along a single dimension. Observers "foraged" for 40 colored target disks that moved randomly across the display. The displays contained targets of two different colors (20 targets per color) among 40 distractors with a different color (see Figure 1 for an example with green and yellow targets among purple distractors; Figure 2 shows another example). Collected targets were replaced so that observers always had plenty of options to pick targets of either color. The actual colors varied from trial to trial, but the relative color space distances between targets and distractor features always adhered to one of three conditions: targets were either close, medium, or far in color distance from each other, and the distractor was always selected from the opposite side of the color circle (see examples in Figure 3). These conditions were presented entirely intermixed.

The main variable of interest was the probability with which observers switched between the two target types. Earlier foraging experiments that looked at target switching tested selection sequences on the trial level against randomness, counting nonrandom trials, and submitting the 
counts to ANOVAs, or they looked at the number or length of same-type runs. This method was able to reveal flexible near-chance switching in a "single-feature" condition and highly reduced switching in a "conjunction” condition (Á. Kristjánsson et al., 2014). However, the questions asked in the present study require a more precise and gradual measure of target switching tendency. We hence used a hierarchical Bayesian model that takes the low-level data distribution (discrete "switch" or "no-switch" events) into account. We estimated the switching probability at the participant and group levels (details can be found in section "Data Analysis"). We expected frequent switching in the "Ts close" condition in which both targets had highly similar colors that could be captured by a single unspecific template (e.g., observers could attend to all yellowish stimuli). For the "Ts medium" and "Ts far" conditions, the two target colors were more distinct, potentially requiring two templates that needed to be switched. Hence, we expected some reluctance to switch in order to avoid switch costs. However, since the distractor is still very far from the targets, and these conditions resembled Á. Kristjánsson et al.'s (2014) feature foraging condition, switching was still likely to be relatively flexible.

We also expected performance decreases if observers are reluctant to switch. As all stimuli were moving randomly, repeated selections of the same target type required observers to either go longer paths (skipping switch targets) or wait longer (until a repeat target moves by), or both. We were interested whether the avoidance of switch costs optimizes overall time performance under the potentially more difficult conditions, or if it is more likely a sign of effort minimization.

\section{Participants}

Thirty-five participants ( 28 female, 7 male) completed the experiment. Their ages ranged from 18 to 34 years $(M=22, S D=3.72)$, and they had normal or corrected-to-normal visual acuity and normal color vision, both tested with the OCULUS Binoptometer3. Even though we did not expect fundamental differences in attentional control in different populations, it should be noted that this was a convenience sample consisting of German university students. The sample size was determined as follows: We used our model (see Section "Data analysis") to estimate the switching probability in Á. Kristjánsson et al.'s (2014) feature foraging condition (the data were publicly available; trials with errors excluded) and obtained $p^{\text {switch }}=.44$ with a $95 \%$ Highest Probability Density (HPD) interval from .4 to .48 . Hence, with the 16 participants in their sample 
the upper HPD limit was smaller than .5 (i.e., nonrandom switching was successfully detected, where $p=.5$ characterizes random switching, given two target categories) and the HPD range was already smaller than .1, showing a good resolution for distinguishing different probabilities. Conservatively, we chose to collect data from about twice as many participants in our experiments.

Participants gave their written consent. The experiment conformed to the ethical standards of the Declaration of Helsinki and was approved by the Ethics Committee of the Faculty of Psychology at Philipps-University Marburg.

\section{Apparatus \& Paradigm}

- "Collect 20 targets"

- 1 point for each target

- 5 points for distractors

- Speed bonus

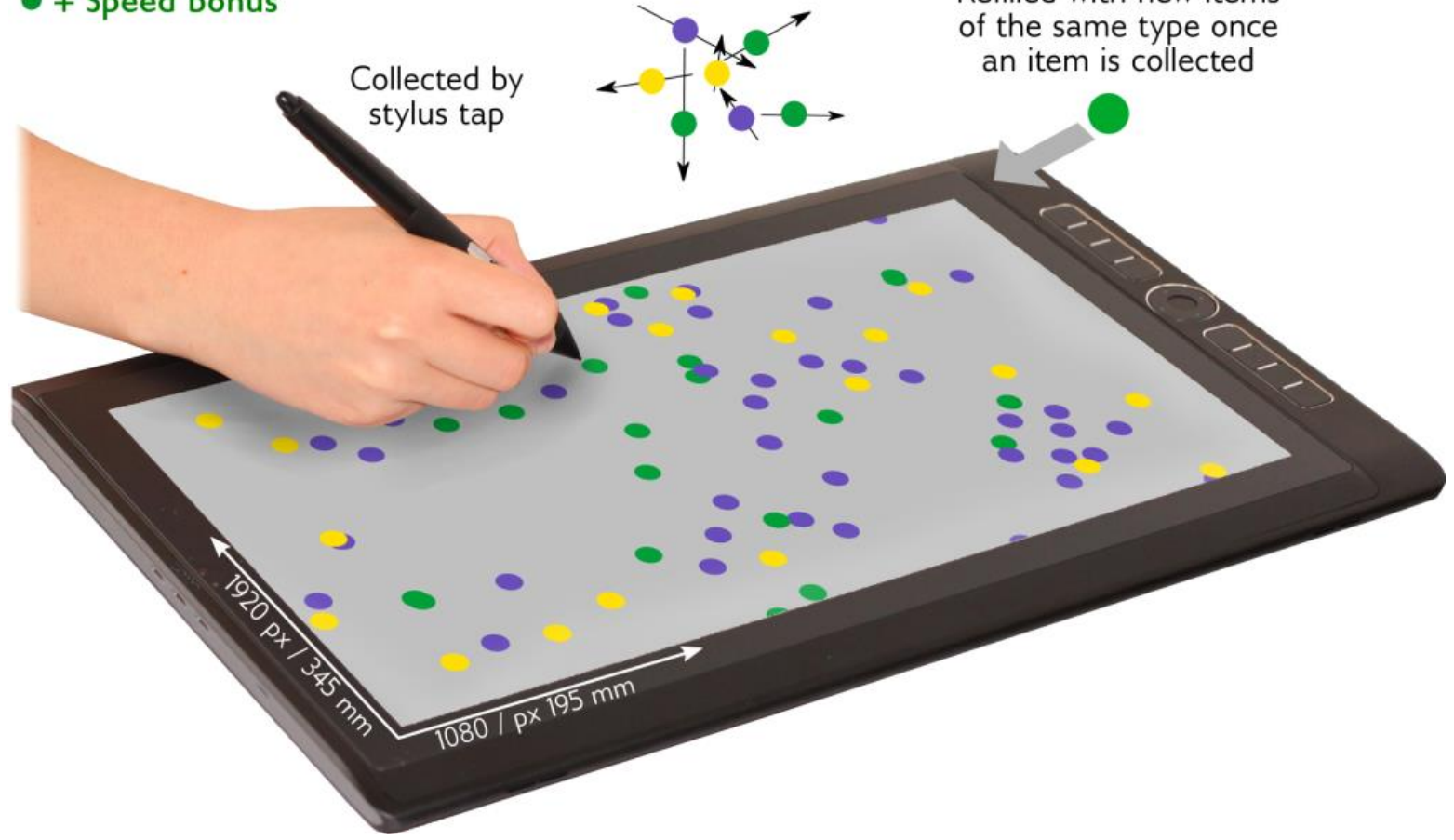

Figure 1. Illustration of the tablet-based foraging task.

The experiment was conducted on a large tablet-PC (WACOM MobileStudio Pro 16 tablet-PC with a display size of $345 \times 195 \mathrm{~mm}$, see Figure 1) at a resolution of $1920 \times 1080$ pixels. It was implemented in OpenSesame (Mathôt et al., 2012) using the PyGame backend for fluent presentation of moving stimuli. In the visual foraging paradigm, participants work through sequences of "patches", displays in which they collect multiple targets among multiple 
distractors (colored disks in this study, see Figure 1). Here, they collected the targets by tapping on them with the tablet-PC's stylus, producing data at a high rate.

The task differed from earlier tablet-based foraging (e.g., Á. Kristjánsson et al., 2014) in two important aspects: (1) The elements were in motion during the trial and (2) once an element was collected, a new element of the same type was pushed into the display at a random location along the screen border with a slight random delay. Since the moving elements also left the display briefly when they reached a border (before they changed their direction and returned), the replacement of collected elements was rather subtle. The motion discouraged strategic selection orders (e.g., in reading direction) which participants sometimes exhibit with static foraging displays (cf. Wolfe et al., 2018; moreover, see Thornton, de’Sperati, \& Kristjánsson, 2019, for evidence that motion does not alter switching behavior).

The replacement of selected elements was motivated as follows: In typical foraging tasks, in which foragers iteratively remove targets from a patch, the constant loss of target options interacts with the tendency to switch between target types or stick with one type in longer runs. When one target type gets rare (or entirely removed), observers are more likely (or have to) switch to the prevalent (or remaining) type. Refilling collected elements disables this interaction. Hence, the switching tendency can be modelled with the relatively simple binomial model of drawing from an urn with replacement. The resulting estimate is a purer reflection of the switching tendency compared to the case in which the collected target type becomes rarer with each selection. Switching probability determined in this manner is a more sensitive and gradual measure than the one-sample runs test typically applied to merely test whether switching is random or nonrandom (e.g., Á. Kristjánsson et al., 2014). Note that unlike other visual foraging studies (e.g., Wolfe, 2013), we were not interested in the relationship between patch leaving and declining picking rates and could therefore replace the collected elements.

\section{Stimuli}

Eighty colored disks served as targets (20 of each target color) and distractors (40 of the distractor color). The disks had a diameter of 48 pixels (approximately $9 \mathrm{~mm}$ ) and were displayed on a gray background $\left(143 \mathrm{~cd} / \mathrm{m}^{2}\right)$. The colors of the targets were taken from angles with $10^{\circ}$ spacing from a color wheel which is part of the CIELAB HLC Colour Atlas (freieFarbe e.V., 2019), and which is illustrated in Figure 3. Note that these colors are not exactly equidistant 
in the CIELAB color space (they have a mean distance of $M=12.74$ units, $S D=4.3$ ), nor was the tablet-PC screen color-calibrated, the luminance matched (it was on average $68 \mathrm{~cd} / \mathrm{m}^{2}, S D=$ 44 ), or individual differences in color perception taken into account. However, as the distance conditions (and in Experiment 2 the relational positions) were realized all over this color wheel (i.e., the exemplary locations depicted in Figure 3 were rotated once to each of all 36 possible positions), we assumed that any nonuniformities average out in the results.

\section{Procedure}

After three practice trials (one of each condition in a randomized order), every participant completed 108 experimental trials. Participants were allowed to collect any mixture of the two target types in any order and were automatically transferred into the next patch after they had collected 40 targets. Between patches, they received point-based feedback. They gained one point for each target they collected and -5 points for each inadvertently collected distractor. If they finished a trial faster than in 45 seconds, they also received a speed bonus of $45-t$, where $t$ is the time required to collect the 40 targets. Participants were shown these details and the overall score.

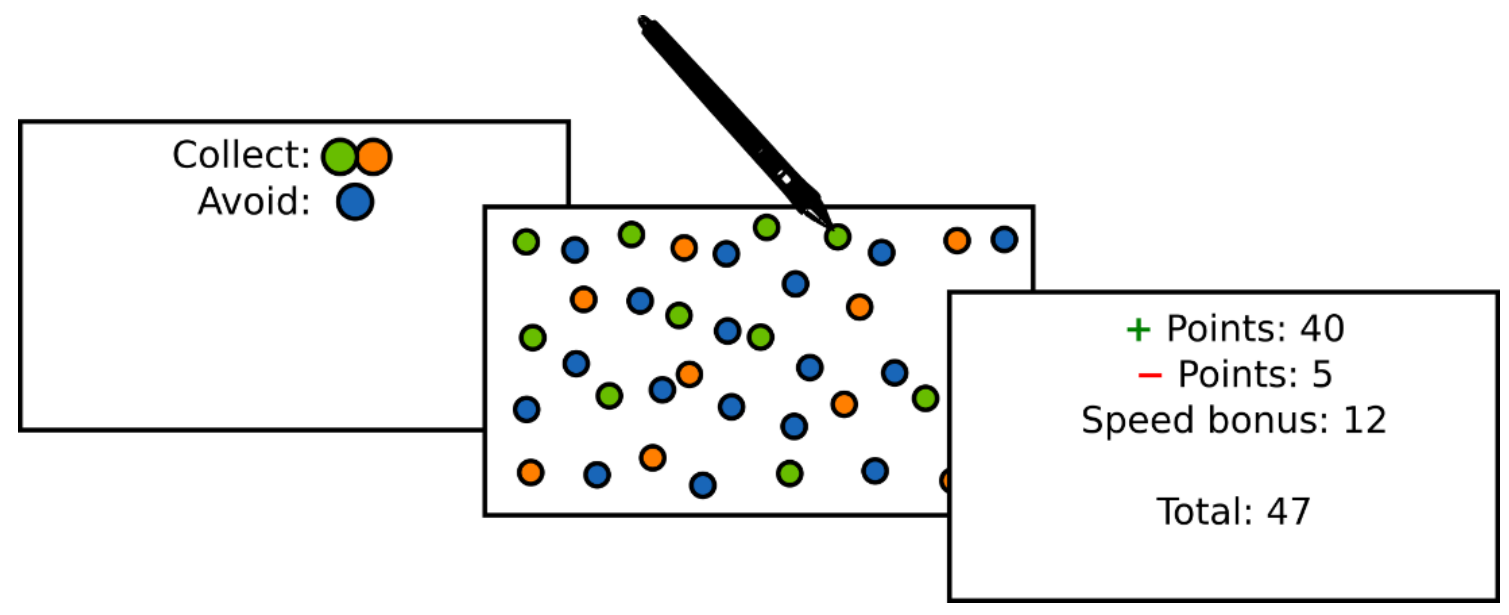

Figure 2: Overview of the procedure: The first display in a trial shows which elements to collect. Then, participants collect 40 of the moving target disks. Afterward, a screen provides performance feedback. Illustration not drawn to scale. Only half the number of disks is shown.

The 108 trials consisted of 36 trials from three conditions, which differed in the color distance between the two targets. To establish the conditions, multiples of the $10^{\circ}$ color steps were used. Each of the 36 colors served as the distractor color once per condition. The target 
colors were selected in the color wheel range opposite to the distractor. In the "Ts close" condition, there was one color step $\left(10^{\circ}\right)$ between the two target colors. In the "Ts medium" and "Ts far" conditions, five color steps $\left(50^{\circ}\right)$ and 11 color steps $\left(110^{\circ}\right)$ separated the targets (see Figure 3 for an illustration). Note that varying the target-target distance in this manner automatically also varies the target-distractor distance. However, the distractor was always so far away from both targets in feature space that it would probably be a categorically different color. Moreover, it is unlikely that the required templates span as far as a third of the color wheel. Hence, it is target-target and not target-distractor similarity what was effectively varied in this experiment.

The trials from all three conditions were presented entirely intermixed. Before each trial started, a screen that showed the two targets and one distractor informed the participants which elements were to be collected and which one to be ignored on a given trial (see Figure 2).

\section{Data Analysis}

The switching data were analyzed with a model using hierarchical Bayesian parameter estimation, which leads to posterior distributions over the likely parameter values (e.g., see Kruschke \& Liddell, 2018). The model is described in detail below. The additional measures (time, distance, errors, switch costs) were assessed with Bayesian versions of common tests such as ANOVAs (provided by JASP, JASP Team, 2020). These tests were executed on average scores for each participant in each condition. Internally, these tests conduct model comparisons and return Bayes factors (BF) that indicate how many times particular "models" (e.g., versions of an ANOVA with or without different combinations of main effects and interactions) are more

likely than a null model. We then report the BF against the null model for the most likely model. Where helpful (when the combinatorial possibilities of model alternatives get large), we report “Analyses of Effects" (cf. van den Bergh et al., 2020; JASP Team, 2020) which provide BFs for the inclusion (or exclusion) of certain effects (e.g., a certain interaction term or main effect). For further details on Bayes factors and their interpretation, see JASP Team (2020) or Kruschke and Liddel (2018).

\section{A Model for Switching Probability}


The main dependent variable, the probability of switching between the two target types, was analyzed via parameter estimation with a hierarchical beta-binomial model (cf. Albert \& $\mathrm{Hu}$, 2019, pp. 381-385):

$$
\begin{aligned}
S_{i, j}^{\text {switch }} & \sim \operatorname{Binomial}\left(p_{i, j}^{\text {switch }}, N\right) \\
p_{i, j}^{\text {switch }} & \sim \operatorname{Beta}\left(\alpha_{i}, \beta_{i}\right) \\
\alpha_{i} & \leftarrow p_{i}^{\text {switch }} N \\
\beta_{i} & \leftarrow\left(1-p_{i}^{\text {switch }}\right) N \\
p_{i}^{\text {switch }} & \sim \operatorname{Beta}(1,1) \\
\Delta p_{k, l}^{\text {switch }} & \leftarrow \operatorname{mean}\left(p_{k, j}^{\text {switch }}-p_{l, j}^{\text {switch }}\right)
\end{aligned}
$$

Variable $S_{i, j}^{\text {switch }}$ refers to the number of switches participant $j$ made in condition $i$, given the $N$ opportunities to switch when collecting $N+1$ elements. Parameter $p_{i, j}^{\text {switch }}$ is the switching probability (of participant $j$ in condition $i$ ). When it is estimated close to .5 , this means that observers switch or stay with a target type at chance level. Smaller values mean that they make runs with targets of the same type longer than expected by chance. When $p_{i, j}^{\text {switch }}$ approaches zero, observers stick with a single target the whole time. Values larger than .5 indicate that observers switch more often than predicted by chance. This means that they intentionally alternate between the target types, a behavior rarely observed for target switching during foraging (cf. Á. Kristjánsson et al., 2014). The Beta(1,1) distribution is a flat prior for the group level, which through transformations (Equations 3 and 4) and the participant level distribution $\operatorname{Beta}\left(\alpha_{i}, \beta_{i}\right)$ assigns equal a priori probabilities to all possible switching probabilities $p_{i, j}^{\text {switch }}$ on the participant level.

This model of target switching was implemented and estimated with PyMC3 (Salvatier et al., 2016). Twenty thousand samples were drawn with the NUTS method (Hoffman \& Gelman, 2014). To assess the target switching probability $p_{i}^{\text {switch }}$, we looked at the mode of its posterior distribution and the $95 \%$ Highest Probability Density (HPD) interval. For statistical comparisons between two conditions (with indices $k$ and $l$ ), we assessed the $95 \%$ HPD of the mean of differences of the switching probability estimates (cf. Equation 6). 


\section{Results}

\section{Target-Switching Probability and Switch Costs}

A

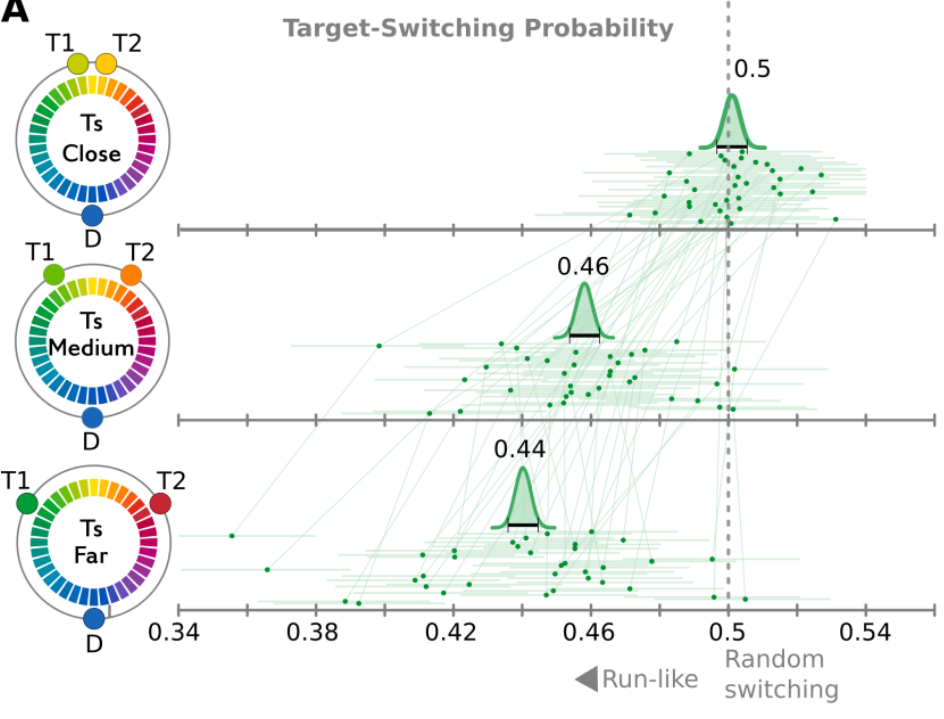

B
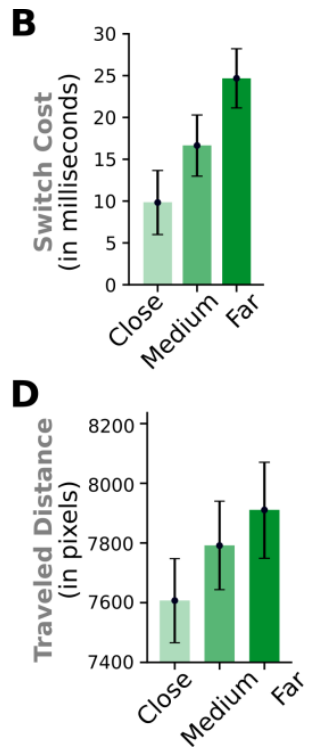

C

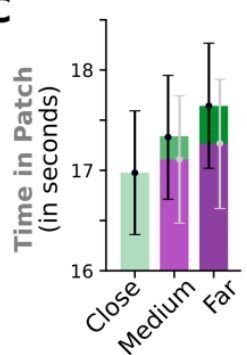

$\mathbf{E}$

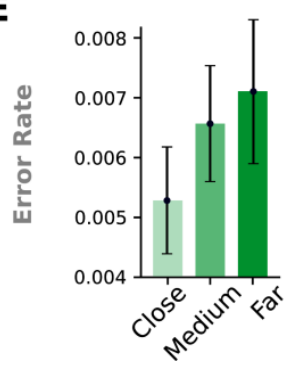

Figure 3: Results of Experiment 1. The colors of T1, T, and D are examples for the respective conditions. During the experiment, the same relative positions were realized all over the color circle. (a) Group-level posterior distributions of the target-switching probabilities; black horizontal ranges indicate the $95 \%$ Highest Probability Density (HPD) intervals. The points below the distributions reflect the estimates for individual participants and the faint horizontal lines are their $95 \%$ HPD intervals. Panels (b) to (e) show the averages of switch costs, overall time spent in a patch, overall distance traveled, and error rate (ranging from 0 to 1). Error bars are $95 \%$ HPD intervals. The purple portions of the "medium" and "far" bars in (c) are predictions for the case "what if foragers would have switched maximally flexibly despite higher switch costs".

The target switching probabilities estimated with the hierarchical Bayesian model are illustrated in Figure 3A. In the "Ts close" condition, target-switching was at chance with .5 (random switching) well within the $95 \%$ HPD interval. For "Ts medium" and "Ts far" distance, switching was below chance, .46 and .44 , respectively, indicating small but systematic tendencies to stick with one of the target types. The overall pattern shows that more distant colors led to reduced target switching. The "Ts medium" condition differs from the "Ts close" condition by .04 [95 \% HPD: .037 to .049], with zero (no difference) far outside the $95 \%$ HPD 
interval. Similarly, "Ts far" differed from "Ts medium" by .018 [95 \% HPD: .012 to .024], again with zero (no difference) well outside the $95 \%$ HPD interval.

Figure 3B shows the switch costs in the different conditions. The switch costs were calculated as the average inter-target time (ITT) from selections in which foragers switched to the other target type minus the average ITT of selections in which the same target was collected (cf. Á Kristjánsson et al., 2019). A Bayesian repeated measures ANOVA with the factor distance returned a Bayes factor of 5.96E+04 in favor for the model with the main effect distance (against the null). Post hoc tests of the pairwise differences yielded Bayes factors > 15, except for the difference between "Ts close" and "Ts medium" which has only moderate support with BF = 3.1.

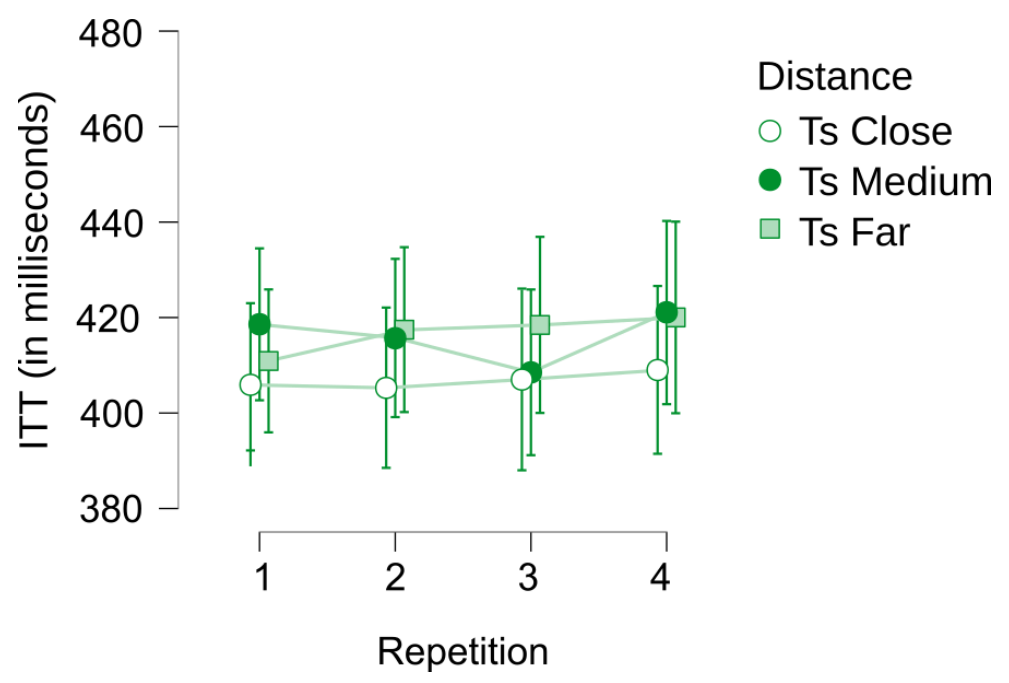

Figure 4. Development of ITTs over repetitions in Experiment 1.

To assess whether ITTs got faster over repeated selections (e.g., due to priming), we extracted selection runs in which the same target was selected two, three, four, or five times (longer runs were increasingly rare) and analyzed the average ITTs before their last selections (see Figure 4). A two-way Bayesian repeated-measures ANOVA was conducted with the factors "Repetitions in Run" and "Color Distance". An Analysis of Effects (cf. van den Bergh et al., 2020; JASP Team, 2020) showed weak evidence in favor of an effect in the color distance condition $\left(\mathrm{BF}_{\text {incl }}=2.78\right)$, and importantly very strong evidence against a repetition effect as well as the interaction of distance and repetition ("Repetitions in Run": $\mathrm{BF}_{\text {excl }}=38.60$, "Repetition in Run" × "Distance": $\left.\mathrm{BF}_{\mathrm{excl}}=54.68\right)$. 


\section{Foraging efficiency: Time in Patch, Traveled Distance, Error Rate}

According to Bayesian repeated measures ANOVAs, the variables "Time in Patch" (TiP; the time required to collect the 40 items; see Figure 3C), "Traveled Distance" (TD; see Figure 3D), "Error Rate" (ER; rate of accidentally selected distractors, see Figure 3E; arcsine corrected for the ANOVA) all showed the effect of color space distance between the targets. The ANOVA models which include this effect are supported by substantial Bayes factors (against the null models): $\mathrm{BF}_{\mathrm{TiP}}=4.18 \mathrm{E}+08, \mathrm{BF}_{\mathrm{TD}}=1.31 \mathrm{E}+09$, and $\mathrm{BF}_{\mathrm{ER}}=47.13$. For "Traveled Distance" and "Time in Patch", post hoc tests (Bayesian $t$ test; JASP Team, 2020) yielded high Bayes factors (all > 90) for all pairwise differences of the different distance conditions. For "Error Rate", only “Ts close" vs. "Ts medium" and "Ts close" vs. "Ts far" led to substantial Bayes factors (19.96 and 43.93, respectively) while there is no statistical support for a difference between "Ts medium" and "Ts far" $(\mathrm{BF}=0.26)$.

\section{Performance Optimization}

We were interested in whether avoiding to switch helped to optimize performance. This question is intricate, because not switching has both advantages and disadvantages: On the one hand, not switching has the advantage of avoiding the delays caused by switching templates (switch costs) but on the other hand it requires observers to go longer distances or wait for other targets moving by, which both cost time (performance costs). Hence, the question boils down to whether the performance we observed in conditions with reduced switching and increased performance costs is shorter than the time expected if observers had switched frequently (to avoid performance costs) despite the frequent and high switch costs.

To this end, we compared the observed "Time in Patch" (TiP) to the predicted time in patches that would be expected (ET) if observers had switched frequently with maximum flexibility. We took the "Ts close" condition as a baseline and added the accumulated switch costs measured for the respective condition. That is, the expected time in patch for the "Ts medium" condition is calculated for each individual as $\mathrm{ET}_{\text {medium }}=\mathrm{TiP}_{\text {close }}+\left(19.5 \cdot\left(\mathrm{SC}_{\text {medium }}-\right.\right.$ $\left.\mathrm{SC}_{\text {close }}\right)$ ), where $\mathrm{TiP}_{\text {close }}$ is the time spent in patches of the "Ts close" condition and 19.5 is the expected average number of switches (in the 39 possibilities to switch) if switching were maximally flexible as seen in the "Ts close" condition). The term $\left(\mathrm{SC}_{\text {medium }}-\mathrm{SC}_{\text {close }}\right)$ is the difference between switch costs in the "Ts medium" and "Ts close" conditions; the term 
$\left(\mathrm{SC}_{\text {medium }}-\mathrm{SC}_{\text {close }}\right)$ replaces the contribution of the very low switch costs to the $\mathrm{TiPc}_{\text {lose }}$, with the higher switch costs from the "Ts medium" condition. The analogous procedure is used to obtain $\mathrm{ET}_{\text {far }}$. As can be seen in the purple portions of the bars in Figure 3C, the overall time in a patch would only slightly increase for the "Ts medium" and "Ts far" conditions if observers switched maximally flexibly despite the higher switch costs. Bayesian $t$ tests, testing for shorter observed than expected times (expected if reduced switching optimizes performance), returned Bayes factors $\leq 0.054$, indicating that it is about 18 times more likely that the observed times in patches were not shorter than the expected ones.

\section{Discussion}

Experiment 1 showed that participants switched target types frequently and with ease in the "Ts close" condition. The switching probability was at chance level, indicating highly flexible switching, and switch costs, around $5 \mathrm{~ms}$, were very small, much smaller than in the other conditions of the experiment. This is well in agreement with the hypothesis that for similar targets with features dissimilar to the distractors, observers can utilize a rather unspecific template that covers a broad range of feature values. In the "Ts medium" and "Ts far" conditions, observers showed moderate reluctance to switch and experienced higher switch costs.

Particularly, in the "Ts far" condition the reluctance to switch and switch costs were the highest. This is in line with the idea that for very different colors, two templates and switching between them is required. In the "Ts medium" condition, switching probability and switch costs fell in between the "Ts close" and "Ts far" results. This could mean that in this condition templates can sometimes be so broad that a template for one target occasionally also selects the other target without causing switch costs. Particularly in some color ranges this seems quite plausible (e.g. in the blueish-greenish or reddish-purplish range).

Concerning the nature of switch costs, the experiment provided two insights. First, there is no evidence that repeated selections are beneficial because of perceptual priming effects. Repeated selection of the same target did not iteratively reduce the inter-target times as happens for RTs in priming experiments (e.g., see Kristjánsson et al., 2002). Instead, the switch costs appear to be a "real" cost and not just the absence of priming. This cost might reflect the operations needed to elevate the other template into the attention guiding state (cf. Olivers at al., 2011). The second insight concerning the nature of switch costs comes from the analysis of the 
actual time required in a patch in relation to the expected time, given observers would have switched more frequently despite increased switch costs. The analysis showed that in the "Ts medium" and "Ts far" conditions, in which there was some reluctance to switch, the actual time in the patch is not shorter than predicted for frequent switching. Indeed, the pattern is the opposite. In these conditions, observers took several hundred milliseconds longer to finish the patches, but the hypothetical accumulated additional switch costs would only make up for fractions of that. We conclude from this that the slight tendencies to stick with the same target type are, in this case, not in the service of performance optimization. It rather seems observers avoid the effort of switching templates, above and beyond its detrimental consequence on time performance. The overall distance traveled to complete a patch and the error rate did also increase with reluctance to switch, indicating that increased time costs are likely not invested to enable short distances while sticking with one target type, or to avoid errors.

All conditions of Experiment 1 allowed for the use of broad templates as the distractor was always far away. Hence, the measurements from Experiment 1 provide a baseline to be compared with the measurement from Experiment 2, in which the feature space proximity of the distractor color disabled the possibility of using broad templates.

\section{Experiment 2}

Experiment 2 tested whether feature contexts with the distractor features close to those of the targets, requiring more specific templates, increase the reluctance to switch between target types and switch costs. Again, the experiment used three intermixed conditions with different feature contexts. In two feature conditions both targets had adjacent hues to each other and to the distractor and were both either to the left or to the right of the distractor hue. We expected this to facilitate narrow attentional templates, as the distractor would interfere with broader ones, but allow relational tuning, that is, shifting the template toward features farther away from the distractor feature. The lowest flexibility in template switching was expected in the third condition, in which the distractor feature was narrowly encompassed by the target features, potentially disabling the possibility of relational strategies. 


\section{Participants}

Thirty-six participants ( 27 female, 9 male) were recruited according to the same criteria as stated for Experiment 1. Participants were between 19 and 30 years old $(M=22.9, S D=2.95)$. All had normal or corrected-to-normal visual acuity and normal color vision (tested with the OCULUS Binoptometer3). Participants gave their written consent, and the experiment adhered to the ethical standards of the Declaration of Helsinki and was approved by the Ethics Committee of the Faculty of Psychology at Philipps-University Marburg.

\section{Apparatus \& Paradigm}

The apparatus and paradigm were the same as in Experiment 1.

\section{Stimuli}

The stimuli were the same as in Experiment 1.

\section{Procedure}

The procedure was the same as in Experiment 1 except for the color space relationships in the three conditions. Instead of looking at close, medium, and far target color distances, this experiment used only close colors (all adjacent colors are separated by a single $10^{\circ}$ step). In two conditions, one target was $10^{\circ}$ and the other $20^{\circ}$ from the distractor, always both in the same direction (to the left in one condition, to the right in the other). In the third condition, the distractor was placed between the two targets (see Figure $5 \mathrm{~A}$ ), hence one target was $10^{\circ}$ to the left and the other $10^{\circ}$ to the right.

\section{Data Analysis}

The same analyses were conducted as for Experiment 1. To additionally test for shifts in tuning functions caused by the relative feature space positions of targets and distractors, the probability of selecting "the rightmost" (or clockwise) target is estimated with the same hierarchical beta-binomial model as used for the switching probability. Instead of the number of switches among the number of possibilities to switch $(N=39)$, the number of selected rightmost targets among all selections $(N=40)$ entered the model for this analysis. 


\section{Results}

Target-Switching Probability and Switch Costs

A

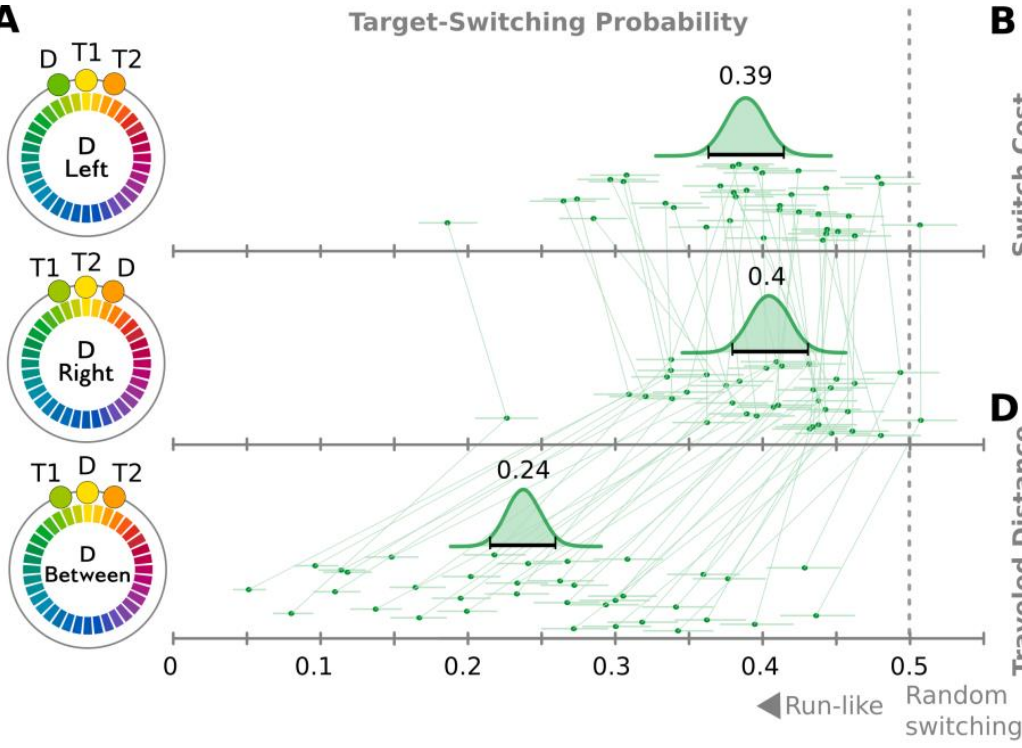

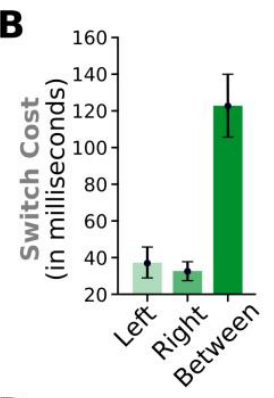

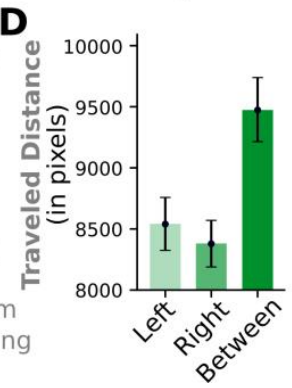

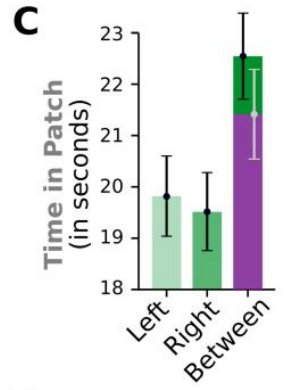

$\mathbf{E}$

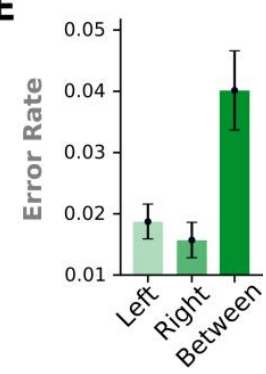

Figure 5: Results of Experiment 2: The colors of T1, T2, and D are examples for the respective conditions. During the experiment, the same relative positions were realized all over the color circle. (a) Group-level posterior distributions of the target-switching probabilities; black horizontal ranges indicate the $95 \%$ Highest Probability Density (HPD) intervals. The points below the distributions reflect the estimates for individual participants and the faint horizontal lines are their $95 \%$ HPD intervals. Panels $(b)$ to $(e)$ show the averages of switch costs, overall time spent in a patch, overall distance traveled, and error rate (ranging from 0 to 1). Error bars are $95 \%$ HPD intervals. The purple portion of the "between" bar in (c) is a prediction for the case "what if foragers would have switched maximally flexibly despite higher switch costs".

Figure 5A depicts the target-switching probabilities estimated with the hierarchical Bayesian model. In the "D left" and "D right" conditions, the probability is close to .4 (.39 and $.4)$, whereas it is .24 in the "D between" condition. All estimates show less switching as predicted by chance (.5 is far outside the $95 \%$ HPD range). The "outside" conditions are rather similar. However, their difference "left minus right" is estimated at -.015 [95 \% HPD: -.021 to -.009], with zero, no difference, just outside the 95\% HPD interval. The "D between" condition is substantially different: The switching probability is reduced by -.155 [95 \% HPD: - 15 to $-.161]$ taking the "right" condition as a reference. 
Concerning the switch costs, the "D between" condition led to strong effects (see Figure 5B): The switch costs in the "D left" and "D right" conditions are between 30 and $40 \mathrm{~ms}$, which is somewhat larger than the highest switch costs observed in Experiment 1. The "D between" condition, however, produces an immense increase to about $120 \mathrm{~ms}$, entering into the range of switch costs which are typically observed with the more difficult conjunction foraging (cf. T. Kristjánsson et al., 2018). A Bayesian repeated measures ANOVA estimated a Bayes factor of $7.88 \mathrm{E}+23$ in favor of the model with the main effect of relative distractor position (against the null). Pairwise post hoc tests produce high Bayes factors for "D left" and "D right" vs. "D between" (both $>1 \mathrm{E}+10)$ but no evidence for a difference between "D left" and "D right" $(\mathrm{BF}=$ $0.34)$.

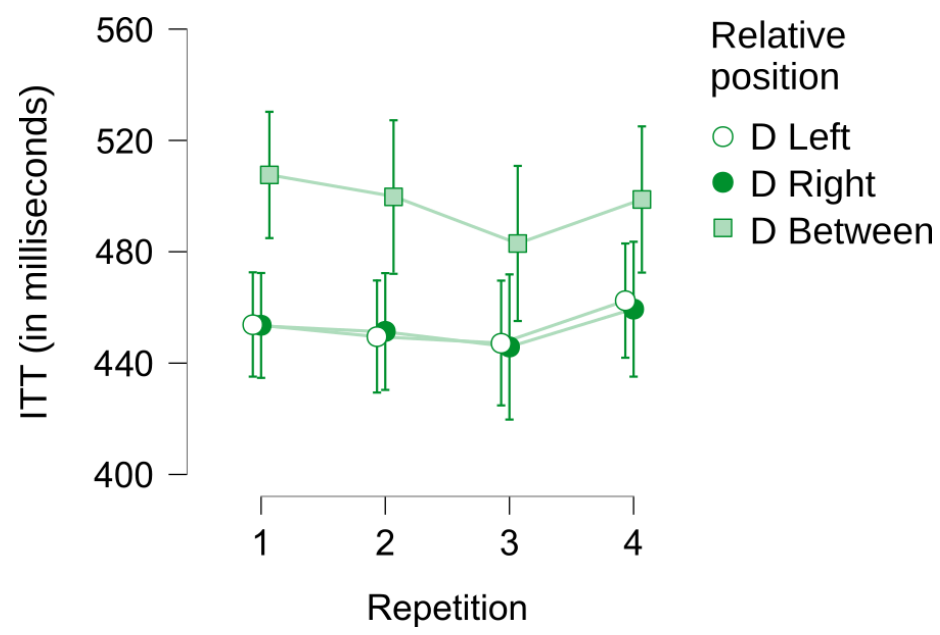

Figure 6. Development of ITTs over repetitions in Experiment 2.

Again, we tested if ITTs decreased as observers repeatedly selected targets of the same type. As for Experiment 1, we extracted all runs with lengths between two and five (1 to 4 repetitions) and tested whether the average ITTs before the last selections in these runs were faster depending on the number of repetitions (cf. Figure 6). Again, a "Repetitions in Run" $X$ "Color Distance" Bayesian repeated-measures ANOVA found no support for priming effects. The Analysis of Effects did reveal strong evidence for a main effect of feature space relationship $\left(B F_{\text {incl }}=1.519 \mathrm{E}+22\right.$; the effect is seen as the offset of the "D between" line in Figure 6). Evidence concerning the priming effect was indecisive (neither favoring inclusion or exclusion, $\mathrm{BF}_{\text {incl }}=0.83$ ). Evidence for the exclusion of the interaction term was strong with $\mathrm{BF}_{\mathrm{excl}}=56.6$. 


\section{Foraging efficiency: Time in Patch, Traveled Distance, Error Rate}

Again, these effects on the switching probability were reflected in the foraging performance parameters: The time in patch (TiP; Figure 5C), traveled distance (TD; Figure 5D), and error rate (ER; Figure 5E) all showed the effect of relative distractor placement, as confirmed by Bayesian repeated measures ANOVAs with the factor relative distractor position: The Bayes factors in favor of the effect (against the null model) are $\mathrm{BF}_{\mathrm{TiP}}=6.98 \mathrm{E}+35, \mathrm{BF}_{\mathrm{TD}}=$ 6.30E+23, and $\mathrm{BF}_{\mathrm{ER}}=9.40 \mathrm{E}+26$. Pairwise post hoc tests of the conditions showed strong evidence in favor of the differences between the "D between" conditions and "D left" and "D right", respectively (all BFs $>1 \mathrm{E}+12$ ). Also, the much smaller difference between "D left" and "D right" has some statistical support. For the error rate, the Bayes factor is very high with 358.1. With regard to "traveled distance" and "time in patch", the Bayes factors for "D left" vs. "D right" are 6.36 and 8.43 .

As in Experiment 1 we looked at whether observers optimized performance and so we again calculated a prediction for the case that observers would have switched maximally flexibly despite the higher costs. This prediction is shown as the purple portion of the "D between" bar in Figure $5 \mathrm{C}$. Note that in this experiment there is no optimal baseline for the time in patch from a condition in which observers switched maximally flexibly. Hence, to obtain an approximation for the prediction, we took the condition with the lowest time in patch ("D right") subtracted the switch costs that occurred in this condition and added those that would have occurred if observers had switched maximally flexibly in the "D between" condition: $\mathrm{ET}_{\text {between }}=\mathrm{TiP}_{\text {right }}$ $\left(N_{\text {right }} \cdot \mathrm{SC}_{\text {right }}\right)+\left(19.5 \cdot \mathrm{SC}_{\text {between }}\right)$, where TiP refers to time in patch, "SC" to switch cost, $\mathrm{N}$ to the number of switches observed, 19.5 to the expected number of switches when observers switch maximally flexibly, and the subscripts indicate the conditions. Subtracting $\left(N_{\text {right }}\right.$. $\left.\mathrm{SC}_{\text {right }}\right)$ removes the switch cost contributions from the observed time in patch, and adding (19.5 $\mathrm{SC}_{\text {between }}$ ) increases the time by the hypothetical accumulated switch costs in the "D between" condition. A Bayesian paired samples t test, testing in the direction of the observed time in patch being smaller than the predicted one, provided no evidence in favor of such a difference, $\mathrm{BF}=$ 0.013 (this corresponds to a BF of 79.67 for the absence of the effect). Hence, there is no indication that avoiding switches improves performance. 


\section{Relational effects}

So far, the analyses reported above were the same as for Experiment 1. However, Experiment 2 required an additional analysis: In contrast to Experiment 1, the two targets were not placed symmetrically with regard to the distractor. Hence, it is possible that observers preferred one of the two targets to implement relational guidance. We tested this by applying the hierarchical beta-binomial model which we also applied to the switch probabilities (see "Data Analysis"). The results of this analysis are shown in Figure 7.

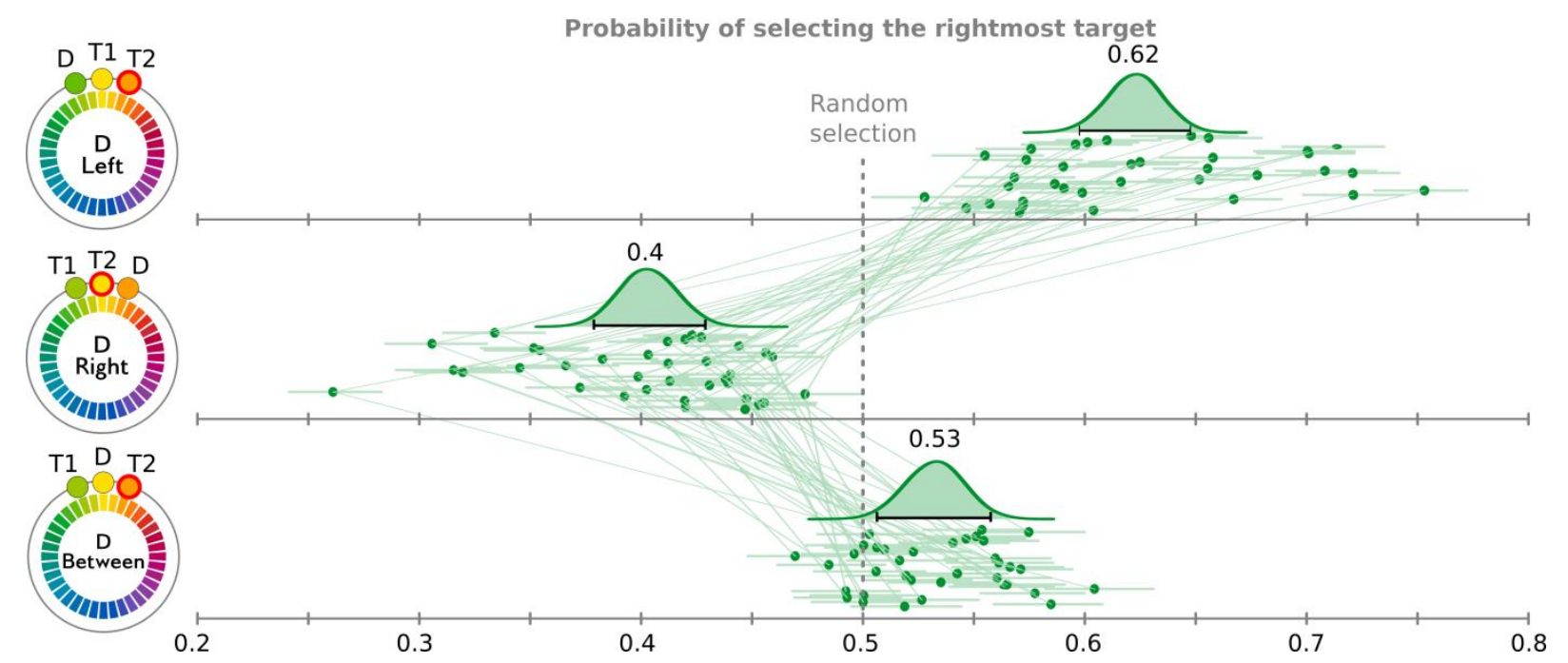

Figure 7: Results of Experiment 2: (a) Group-level posterior distributions for the probability of selecting the "rightmost" (clockwise) target (marked by a red circle) in the three different conditions. The concrete colors in the illustration at the left of each row are only exemplary instances. During the experiment, the same relative positions were realized all over the color circle. Black horizontal ranges indicate the $95 \%$ Highest Probability Density (HPD) intervals. The points below the distributions reflect the estimates for individual participants and the faint horizontal lines are their $95 \%$ HPD intervals.

In the "D left" condition (Fig. 7, upper row), the rightmost target, which is the one farther away from the distractor, was predominantly selected (probability .62, the balanced selection probability of .5 is far outside the 95\% HPD interval). For the "D right" condition (Fig. 7, middle row), the probability of selecting the rightmost target is only .4 (balanced selection probability of .5 is far outside the 95\% HPD interval). This means the target closer to the distractor is selected less often, or conversely, the one farther away is selected more often, in 
agreement with the former "D left" condition. For the "D between" condition, the .53 probability of selecting the rightmost target is relatively close to balanced selection at .5 . However, .5 is slightly outside the 95\% HPD interval. Hence, the shift of the distribution might indicate a slight rightward (clockwise) color selection bias. The almost centered distribution indicates that observers have no strong bias towards either target across trials. Note that observers could still tend to pick one of the targets in a given trial. This is even expected for observers with strongly run-like behavior (e.g., if someone forages in a run with length 30, unavoidably there is a 30 out of 40 bias in that trial). Hence, the nearly centered estimate for the between condition does not rule out biases towards one of the targets within any given trial, instead it indicates that there is no strong rightward or leftward hue bias across trials.

\section{Comparing Experiment 1 and 2}

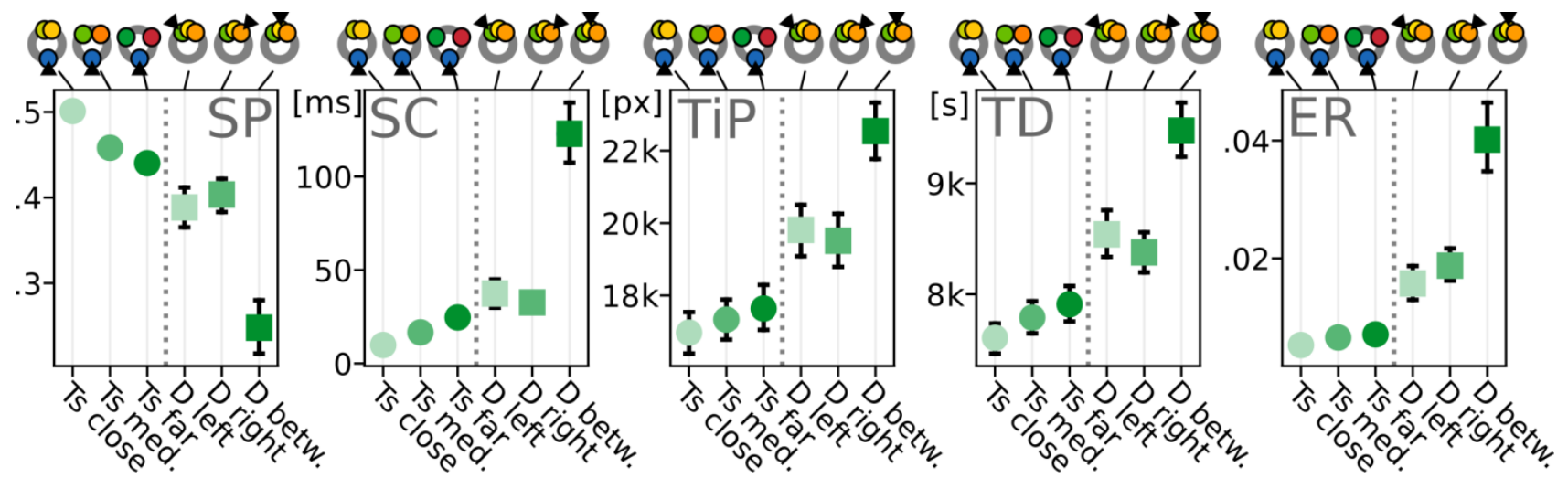

Figure 8. Comparison between the conditions and experiments for switching probability (SP), switch costs (SC), Time in Patch (TiP), Traveled Distance (TD), and Error Rate (ER). Disks represent conditions from Experiment 1, squares represent conditions from Experiment 2. The icons in the top line illustrate instances of the conditions (not drawn to feature space scale). Error bars are $95 \%$ Highest Probability Density intervals and are omitted if smaller than the markers (see Figures 3 \& 5 for clearer representations of certainty).

In Experiment 2, the distractor color was highly similar to the target colors, requiring templates much more specific than in Experiment 1. To test whether this led to the expected increase in switching reluctance we compared the switching probabilities between experiments. When comparing Experiments 1 and 2 (see Figure 8), it is important to note that even though Experiment 2's "D left" and "D right" conditions led to larger switching probabilities than its "D 
between" condition, all of Experiment 2's conditions led to smaller probabilities than Experiment 1 's "far" condition (and the other conditions of Experiment 1, which had even higher switching probabilities): The differences between the group-level estimates $p_{\text {far }}^{\text {switch }}$ (Experiment 1) and $p_{\text {left }}^{\text {switch }}$ (Experiment 2) were estimated at .052 [95\% HPD: .017 to 0.09]. For $p_{\text {far }}^{\text {switch }}$

(Experiment 1) and $p_{\text {right }}^{\text {switch }}$ (Experiment 2), the difference was .036 [95\% HPD: 0 to 0.073].

Switch costs and parameters of foraging efficiency mirror this pattern of differences between the experiments: Even in Experiment2's relatively flexible "D left" and "D right" conditions, foraging was less efficient than in Experiment 1's least efficient condition ("far"), as revealed by Bayesian independent sample $t$ tests with $\mathrm{BF}_{\mathrm{SC}}>3, \mathrm{BF}_{\mathrm{TD}}>95, \mathrm{BF}_{\mathrm{TiP}}>492$, and $\mathrm{BF}_{\mathrm{ER}}>1.16 \mathrm{E}+05$.

\section{Discussion}

In Experiment 2 observers were more reluctant to switch than in Experiment 1, experienced higher switch costs, and faced a stronger impact on parameters of foraging efficiency (time in patch, distance traveled, error rate), see Figure 8. This supports the hypothesis that the more specific attentional templates required by the feature-space proximity of the distractor made template switching more demanding. The highest reluctance to switch and largest impact on performance occurred in the condition in which relational strategies were discouraged whereas feature specific ones were encouraged by placing the distractor between the targets. This finding is in agreement with the hypothesis that the highly specific template is the costliest one to switch. Note that we cannot entirely rule out relational strategies in this condition. Observers might focus on one of the targets and redefine all other stimuli as nontargets. This would have been and fully admissible strategy to solve the task. From the almost centered participant level estimates in Figure 7 (lowest row) we know that if observers used such strategies, they did so with no consistent tendency in one or the other feature space direction. Nevertheless, even if observers would have implemented relational search in this manner, this condition would still require the most specific template in the experiment. In the "D left" and "D right" condition observers could have shifted their templates farther away from the distractor feature and made them broader (and still reaching the outer target) than in the "D between" condition. Moreover, if templates are asymmetrically sharpened as suggested by Yu \& Geng (2019), a sharper, more specifically shaped template would be necessary in the "D between" 
condition. Hence, the result patterns between Experiments 1 and 2 and within Experiment 2 show that reluctance to switch templates increases with template specificity.

Regarding the nature of switch costs, there was again no evidence for facilitation due to priming and hence switch costs were not the absence of priming benefits but true disadvantages associated with target switching. Moreover, as in Experiment 1 the observed time performance (time in patch) in the condition with the lowest switching tendency was not better than the one predicted for the case in which observers switch maximally flexibly despite higher switch costs. "Observed better than predicted" would have been expected if observers avoided switching to maximize performance. Consequently, performance optimization seems not the reason for reluctance to switch and instead observers seem to avoid the cognitive effort of template switching.

\section{General Discussion}

In the present study we observed highly informative differences in foraging behavior in feature conditions, in which stimuli differed along a single dimension. Earlier research regarded foraging for targets defined by a single feature as highly flexible with negligible switch costs (e.g., T. Kristjánsson et al., 2019). While our findings confirm this for well-separated target and nontarget features, they also show that under less favorable conditions switching is costly and reduced to levels typical for more complex targets (Á. Kristjánsson, et al. 2019; Wolfe et al., 2016). The overall pattern shows increased reluctance to switch and higher switch costs in feature contexts that require more specific attentional templates, supporting the hypothesis that effort increases with template specificity. This is in line with and elaborates on earlier observations that searchers can implement highly specific attentional control strategies but often refrain from it (Bacon \& Egeth, 1994, Becker et al., 2014; Yu \& Geng, 2019).

Perhaps most strikingly, the least switching occurred when relational strategies (e.g., selecting the "redder" elements) were discouraged and a highly feature-specific template was required. Parameters of foraging performance (distance, time, and errors) showed consistent efficiency decreases with increasing reluctance to switch. This alone does not rule out that observers reduce switches to increase the overall performance. It could be well possible that they would be worse off when switching more frequently, because of the higher switch costs in the more demanding conditions. However, for both experiments, projections of the expected time 
performance for frequent switching in the conditions with higher switch costs revealed that the observed behavior was not more efficient. Hence, mental effort might be the reason for reduced switching. This agrees with findings in the adaptive-choice visual search literature, which link delays in updating attentional control settings to effort minimization (Irons \& Leber, 2016; 2018; Bergmann et al., 2019). Also, earlier foraging studies showed that observers can switch more frequently than what might be their default mode. Thornton et al. (2020) showed that if pressed to forage within certain time constraints, foragers were less reluctant to switch between target types. This goes well with the idea that foragers reduce mental effort (despite performance costs) if they can, but can also forage more flexibly (at the cost of encountering more effort) if they have to.

Ort and Olivers (2020) proposed a taxonomy with three potential stages at which switch costs could occur: A preparation stage, at which templates are initiated, a selection stage, at which the templates interact with the incoming visual information (in order facilitating selection of relevant information), and a post-selection processing stage, at which selected items are confirmed as targets or rejected. The present study provides some insight into the origin of switch costs. Switching was avoided if more specific templates were needed, which was determined by the overall feature context in the display. The template specialization process is perhaps best accommodated in the "preparation stage", because once modified, templates could be repeatedly used for selection and confirmation, as long as observers search for the same target type. Additional modulation seems to occur in the "selection stage": When broad templates cover both target types, observers could forage for them without actually switching the template. Resulting switches would be essentially cost-free, enabling highly flexible foraging as in Experiment 1's "Ts close" condition. 

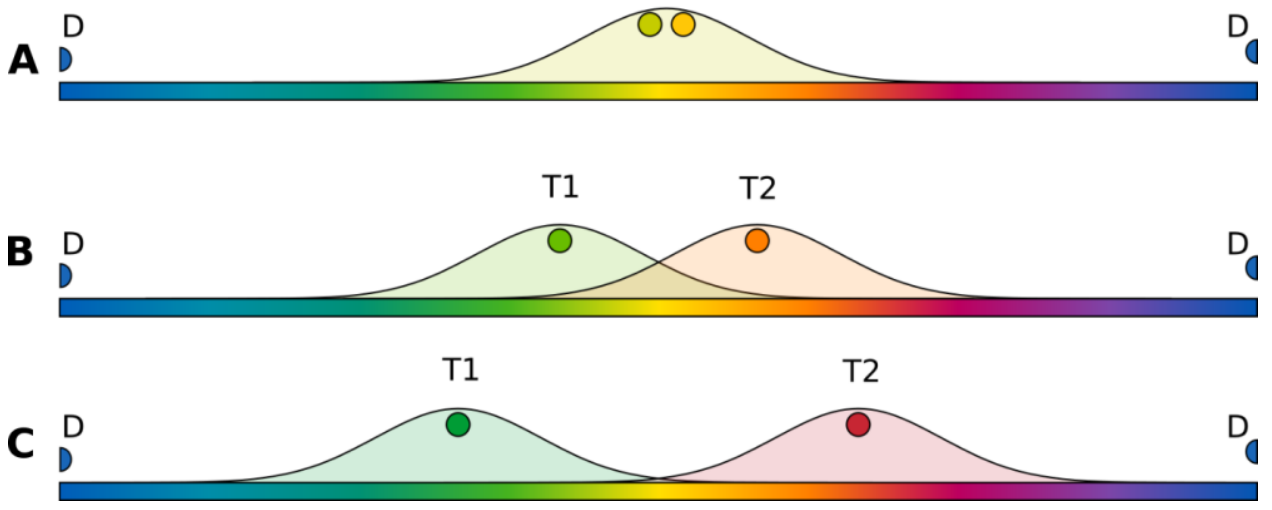

Figure 9. Hypothetical tuning functions for Experiment 1. (a) "Ts close”, (b) "Ts medium”, (c) "Ts far". The "T1" and "T2" indicate target feature space positions, "D" marks the distractor (shown as two halves in this straightened version of the color circle). As with Figures 3, 5 and 7, illustrated colors are examples of the relationships and distances of interest.

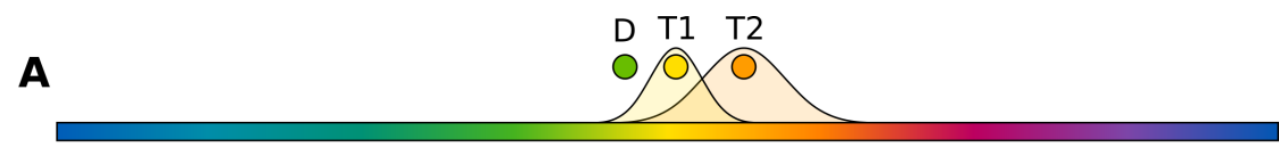

T1 T2 D

B
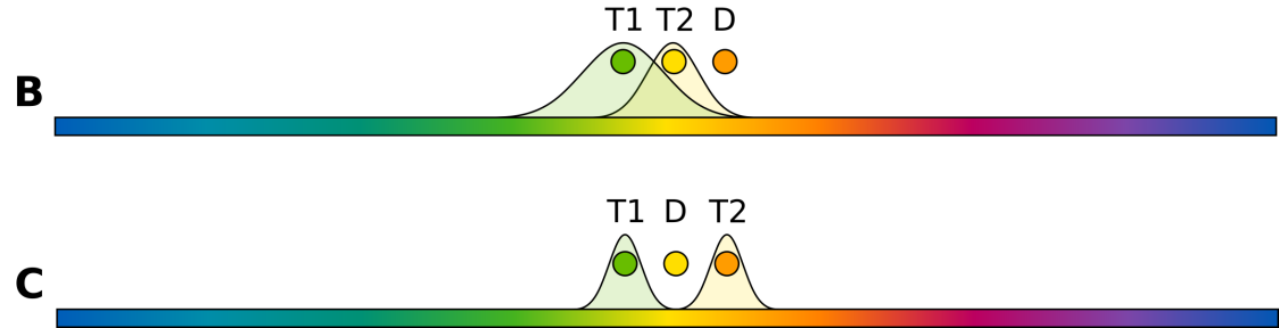

Figure 10. Hypothetical tuning functions as in Figure 9 but for Experiment 2. (a) "D left”, (b) "D right”, (c) "D between”.

On an implementational level, our data might be explained with tunable low-level filters, which depend on the condition and are influenced by one or more templates. If target colors are dissimilar or if the distractor feature is so close in feature space that target templates must be fine-grained, each template requires its own tuning function (Figure 9B \& C, Figure 10). Switching targets then requires unbinding the function from one template and rebinding it to the other in the corresponding feature range. Managing narrow filters is time consuming and foragers avoid switching when possible. Highly similar targets can be efficiently covered with a single tuning function (Figure 9A). In this view, single-template limitations seem rooted not in VWM but in the low-level tuning function, which cannot simultaneously facilitate nonadjacent 
feature values. Observers can multiplex different tuning functions but they avoid it if the task requires costly narrow filters.

The present study also makes contributions to the ongoing discussion of whether only a single VWM representation or multiple ones can guide attention at the same time. Reluctance to switch and switch costs indicate that a single representation is in an attention-guiding state as suggested by Olivers et al. (2011; SIT; single-item-template). However, the ease with which the attention guiding representation is switched seems to depend on the feature context and the specificity of the template. Especially when templates are rather coarse, the system might multiplex between different templates so quickly and effortlessly that it might appear as if multiple templates guide attention simultaneously (MIT; multi-item-template).

This might explain conflicting findings in the literature that favor SIT (e.g., Frătescu et al., 2019, 2014; Olivers et al., 2011) or MIT (e.g., Kerzel \& Witzel, 2019; Hollingworth and Beck, 2016, Frătescu et al., 2019). For instance, rather similar experiments by Van Moorselaar et al. (2014) and Hollingworth and Beck (2016) supported SIT and MIT, respectively (both experiments and their conflicting results were later replicated by Frătescu et al., 2019). In each trial of van Moorselaar et al.'s (2014) experiment, observers memorized one or two colors from a memory display and then searched for a shape singleton in a search display, a subsequent memory test ensured that items were remembered. If one color was remembered, an additional matching color singleton caused strong distraction. If two colors were memorized, the distraction was weaker than with one color. The authors concluded that only a single and not two memory items would lead to an attentional template that interfered with search. Hollingworth and Beck's (2016) task was similar but observers always had to remember two colors, and participants searched for a rotated U-shape singleton. Most of the search displays contained two distractors which could be unrelated to the memory colors, one of them matching, or both matching. The authors found increasing distraction depending on the number of matching distractors and concluded that both memory colors led to templates that could affect the search.

Although speculative, the present findings might help to explain the results of both studies as follows: In van Moorselaar et al.'s (2014) study, with a single colored element in the memory display, the system might be cued to set up (and maintain without effort) a color-based template that interferes with the search. When there are two colors in the memory display, the system would need to decide for one of them, or to start the effortful multiplexing mode. With 
only a single color in the search displays, there might not have been sufficient motivation to activate the effortful multiplexing mode for two templates. In Hollingworth and Beck's (2016) task, most of the displays contained two colors. These multicolored displays might have fooled the system into assuming that color is relevant for search, justifying the multiplexing mode. Visual foraging paradigms as the one used in the present study might provide a more direct route to study attentional templates and can perhaps provide further insights in the future.

The present study was limited to a single feature dimension (hue), leaving open the question whether the findings transfer to other dimensions (e.g., intensity or orientation), multidimensional feature spaces, or natural objects. Real-world objects are rarely defined by single features and our findings might not necessarily generalize to many everyday tasks. However, in addition to illuminating some of the mechanics of attentional control, they can be instructive for designing efficient visualizations especially for the foraging-like tasks performed by, for example, baggage screeners and radiologists (cf. Wolfe, 2016). Interface designers should avoid configurations that require narrow attentional filters (rendering attentional control less flexible) and facilitate the use of efficient relational search strategies.

\section{Open Practices Statement}

Neither of the experiments reported in this article was formally preregistered. The data of both experiments have been made available on https://osf.io/as273/. Moreover, the full model comparison tables of the Bayesian tests can be found in the repository. Requests for further materials can be sent via email to the lead author at jan.tuennermann@ uni-marburg.de.

\section{Author Contributions}

J. T. and A. S. developed the study concept and the design of Experiment 1. J. T., A. S., and L. C. contributed to the design of Experiment 2. Testing, data collection and analysis was performed by J. T. All authors interpreted the results. J. T. drafted the manuscript, and A. S. and L. C. provided critical revisions. All authors approved the final version of the manuscript for submission.

\section{Acknowledgements}

We thank Árni Kristjánsson for helpful comments on an earlier version of the manuscript. 


\section{References}

Albert, J., \& Hu, J. (2020). Probability and Bayesian modeling. Chapman \& Hall/CRC. https://doi.org/10.1201/9781351030144

Becker, S. I. (2010). The role of target-distractor relationships in guiding attention and the eyes in visual search. Journal of Experimental Psychology: General 139(2), 247-265. https://doi.org/10.1037/a0018808

Becker, S. I., Harris, A. M., Venini, D., \& Retell, J. D. (2014). Visual search for color and shape: When is the gaze guided by feature relationships, when by feature values? Journal of Experimental Psychology: Human Perception and Performance, 40(1), 264-291. https://doi.org/10.1037/a0033489

Bacon, W. F., \& Egeth, H. E. (1994). Overriding stimulus-driven attentional capture. Perception \& Psychophysics, 55(5), 485-496. https://doi.org/10.3758/BF03205306

Bergmann, N., Tünnermann, J. \& Schubö, A. (2019). Which search are you on? Adapting to color while searching for shape. Attention Perception Psychophysics, 82(2), 457-477. https://doi.org/10.3758/s13414-019-01858-6

Carlisle, N. B., Arita, J. T., Pardo, D., \& Woodman, G. F. (2011). Attentional Templates in Visual Working Memory. Journal of Neuroscience, 31(25), 9315-9322. https://doi.org/10.1523/JNEUROSCI.1097-11.2011

Chelazzi, L., Miller, E. K., Duncan, J., \& Desimone, R. (1993). A neural basis for visual search in inferior temporal cortex. Nature, 363, 345-347. https://doi.org/10.1038/363345a0

Chelazzi, L., Duncan, J., Miller, E. K., \& Desimone, R. (1998). Responses of Neurons in Inferior Temporal Cortex During Memory-Guided Visual Search. Journal of Neurophysiology, 80(6), 2918-2940. https://doi.org/10.1152/jn.1998.80.6.2918

Duncan, J., \& Humphreys, G. W. (1989). Visual search and stimulus similarity. Psychological Review, 96(3), 433-458. https://doi.org/10.1037/0033-295X.96.3

Frătescu, M., van Moorselaar, D., \& Mathôt, S. (2019). Can you have multiple attentional templates? Large-scale replications of Van Moorselaar, Theeuwes, and Olivers (2014) and Hollingworth and Beck (2016). Attention, Perception, \& Psychophysics, 81(8), 27002709. https://doi.org/10.3758/s13414-019-01791-8

freieFarbe e.V. (2019). CIELAB HLC Colour Atlas XL package (Version 1.2) [Computer software]. 
Geng, J. J., DiQuattro, N. E., \& Helm, J. (2017). Distractor probability changes the shape of the attentional template. Journal of Experimental Psychology: Human Perception and Performance, 43(12), 1993-2007. https://doi.org/10.1037/xhp0000430

Geng, J. J., \& Witkowski, P. (2019). Template-to-distractor distinctiveness regulates visual search efficiency. Current Opinion in Psychology, 29, 119-125. https://doi.org/10.1016/j.copsyc.2019.01.003

Hoffman, M. D., \& Gelman, A. (2014). The No-U-turn sampler: Adaptively setting path lengths in Hamiltonian Monte Carlo. Journal of Machine Learning Research, 15(47), 15931623. https://dl.acm.org/doi/10.5555/2627435.2638586

Hollingworth, A., \& Beck, V. M. (2016). Memory-based attention capture when multiple items are maintained in visual working memory. Journal of Experimental Psychology: Human Perception and Performance, 42(7), 911-917. https://doi.org/10.1037/xhp0000230

Hommel, B. (2009). Action control according to TEC (theory of event coding). Psychological Research PRPF, 73, 512-526. https://doi.org/10.1007/s00426-009-0234-2

Irons, J. L., \& Leber, A. B. (2016). Choosing attentional control settings in a dynamically changing environment. Attention, Perception \& Psychophysics, 78(7), 2031-2048. https://doi.org/10.3758/s13414-016-1125-4

Irons, J. L., \& Leber, A. B. (2018). Characterizing individual variation in the strategic use of attentional control. Journal of Experimental Psychology: Human Perception and Performance, 44(10), 1637-1654. https://doi.org/10.1037/xhp0000560

JASP Team (2020). JASP (Version 0.13.1) [Computer software].

Kerzel, D. (2020). Direct evidence for the optimal tuning of attention. Journal of Experimental Psychology: Human Perception and Performance, 46(7), 716-728. https://doi.org/10.1037/xhp0000744

Kerzel, D., \& Witzel, C. (2019). The allocation of resources in visual working memory and multiple attentional templates. Journal of Experimental Psychology: Human Perception and Performance, 45(5), 645-658. https://doi.org/10.1037/xhp0000637

Kristjánsson, Á., Jóhannesson, Ó. I., \& Thornton, I. M. (2014). Common attentional constraints in visual foraging. PloS ONE, 9(6), Article e100752. https://doi.org/10.1371/journal.pone.0100752 
Kristjánsson Á., Ólafsdóttir I.M., Kristjánsson T. (2019) Visual foraging tasks provide new insights into the orienting of visual attention: Methodological considerations. In S. Pollmann (Ed.), Spatial learning and attention guidance (Neuromethods, vol 151, pp. 321). Humana. https://doi.org/10.1007/7657_2019_21

Kristjánsson, Á., Wang, D., \& Nakayama, K. (2002). The role of priming in conjunctive visual search. Cognition, 85(1), 37-52. https://doi.org/10.1016/S0010-0277(02)00074-4

Kristjánsson, T., Thornton, I. M., Chetverikov, A., \& Kristjánsson, Á. (2020). Dynamics of visual attention revealed in foraging tasks. Cognition, 194, 104032. https://doi.org/10.1016/j.cognition.2019.104032

Kristjánsson, T., Thornton, I. M., Kristjánsson, Á. (2018). Time limits during visual foraging reveal flexible working memory templates. Journal of Experimental Psychology: Human Perception and Performance 44(6), 827-835. https://doi.org/10.1037/xhp0000517

Kruschke, J.K., Liddell, T.M. (2018). Bayesian data analysis for newcomers. Psychonomic Bulletin \& Review, 25, 155-177. https://doi.org/10.3758/s13423-017-1272-1

Martin, A., \& Becker, S. I. (2018). How feature relationships influence attention and awareness: Evidence from eye movements and EEG. Journal of Experimental Psychology: Human Perception and Performance, 44(12), 1865-1883. http://dx.doi.org/10.1037/xhp0000574

Martinez-Trujillo, J. C., \& Treue, S. (2004). Feature-based attention increases the selectivity of population responses in primate visual cortex. Current Biology, 14(9), 744-751. https://doi.org/10.1016/j.cub.2004.04.028

Mathôt, S., Schreij, D., \& Theeuwes, J. (2012). OpenSesame: An open-source, graphical experiment builder for the social sciences. Behavior Research Methods, 44(2), 314-324. https://doi.org/10.3758/s13428-011-0168-7

Navalpakkam, V., Itti, L. (2007). Search goal tunes visual features optimally. Neuron, 53, 605617. https://doi.org/10.1016/j.neuron.2007.01.018

Olivers, C. N., Peters, J., Houtkamp, R., \& Roelfsema, P. R. (2011). Different states in visual working memory: When it guides attention and when it does not. Trends in Cognitive Sciences, 15(7), 327-334. https://doi.org/10.1016/j.tics.2011.05.004

Olivers, C. N., Meijer, F., \& Theeuwes, J. (2006). Feature-based memory-driven attentional capture: visual working memory content affects visual attention. Journal of Experimental 
Psychology: Human Perception and Performance, 32(5), 1243-1265. https://doi.org/10.1037/0096-1523.32.5.1243

Ort, E., Olivers, C. (2020). The capacity of multiple-target search. Visual Cognition, 27(5-8), 330-355. https://doi.org/10.1080/13506285.2020.1772430

Salvatier, J., Wiecki, T. V., \& Fonnesbeck, C. (2016). Probabilistic programming in Python using PyMC3. PeerJ Computer Science, 2, Article e55. https://doi.org/10.7717/peerj$\underline{\mathrm{cs} .55}$

Thornton, I. M., de'Sperati, \& C., Kristjánsson, Á. (2019). The influence of selection modality, display dynamics and error feedback on patterns of human foraging. Visual Cognition, 27(5-8), 626-648. https://doi.org/10.1080/13506285.2019.1658001

Thornton, I. M., Nguyen, T. T., \& Kristjánsson, Á. (2020). Foraging tempo: Human run patterns in multiple-target search are constrained by the rate of successive responses. Quarterly Journal of Experimental Psychology. https://doi.org/10.1177/1747021820961640

van den Bergh, D., van Doorn, J., Marsman, M., Draws, T., van Kesteren, E., Derks, K. \& Wagenmakers, E. (2020). A Tutorial on Conducting and Interpreting a Bayesian ANOVA in JASP. L'Année psychologique, 1(1), 73-96. https://doi.org/10.3917/anpsy1.201.0073

van Moorselaar, D., Theeuwes, J., \& Olivers, C. N. (2014). In competition for the attentional template: Can multiple items within visual working memory guide attention? Journal of Experimental Psychology: Human Perception and Performance, 40(4), 1450-1464. https://doi.org/10.1037/a0036229

Wolfe, J. M. (2013). When is it time to move to the next raspberry bush? Foraging rules in human visual search. Journal of Vision, 13(3), 1-10. https://doi.org/10.1167/13.3.10

Wolfe, J. M. (2016). Use-inspired basic research in medical image perception. Cognitive Research: Principles and Implications, 1(1), 17. https://doi.org/10.1186/s41235-016-0019-2 Wolfe, J. M., Aizenman, A. M., Boettcher, S. E., \& Cain, M. S. (2016). Hybrid foraging search: Searching for multiple instances of multiple types of target. Vision Research, 119, 50-59. https://doi.org/10.1016/j.visres.2015.12.006

Wolfe, J. M., Cain, M. S., \& Alaoui-Soce, A. (2018). Hybrid value foraging: How the value of targets shapes human foraging behavior. Attention, Perception, \& Psychophysics, 80, 609-621. https://doi.org/10.3758/s13414-017-1471-X 
Wolfe, J. M., \& Horowitz, T. S. (2017). Five factors that guide attention in visual search. Nature Human Behaviour, 1, 0058. https://doi.org/10.1038/s41562-017-0058

York, A., Becker, S. I. (2020). Top-down modulation of gaze capture: Feature similarity, optimal tuning, or tuning to relative features? Journal of Vision, 20(4), 1-16. https://doi.org/10.1167/jov.20.4.6

Yu, X., \& Geng, J. (2019). The attentional template is shifted and asymmetrically sharpened by distractor context. Journal of Experimental Psychology: Human Perception and Performance, 45(3), 336-353. https://doi.org/10.1037/xhp0000609 